\title{
Moderating Perceptions of Bother Reports by Individuals Experiencing Lower Urinary Tract Symptoms
}

\author{
Elizabeth M. Botelho' ${ }^{1}$, Emily A. Elstad ${ }^{2}$, Simone P. Taubenberger ${ }^{1}$, and Sharon L. \\ Tennstedt ${ }^{1}$ \\ ${ }^{1}$ New England Research Institutes, Watertown, Massachusetts, USA \\ 2University of North Carolina at Chapel Hill, Chapel Hill, North Carolina, USA
}

\section{Abstract}

We compared reports of symptom bother for the same urinary symptoms to understand why symptom severity and bother do not correspond in a straightforward manner. We used a grounded theory approach to analyze qualitative data from 123 individual interviews and developed a conceptual framework, identifying three symptom perceptions that might "moderate" symptom bother: causal, relative, and uncertainty. Symptom bother was lower for respondents who viewed symptoms causally (symptoms seemed explainable or "normal") or relatively (urinary symptoms were compared to other symptoms or conditions). Bother tended to be higher for respondents who viewed symptoms with uncertainty (when symptom etiology and course were unknown). A greater portion of respondents in the causal perception group had not sought health care for their symptoms. This conceptual framework is useful for understanding the relationship between reactions to and health care-seeking for other symptoms.

\section{Keywords}

community and public health; health seeking; interviews; semistructured; qualitative analysis; sensitive topics

\begin{abstract}
Lower urinary tract symptoms (LUTS) are common among men and women (Herschorn, Gajewski, Schultz \& Corcos, 2008; Kaplan et al., 2009; Kupelian et al., 2006; Pinnock \& Marshall, 1997), yet numerous researchers have found that many persons with these symptoms fail to seek health care (Andersson, Johansson, Sahlberg-Blom, Pettersson \& Nilsson, 2005; Brittain, Perry, \& Williams, 2001; Shaw, Tansey, Jackson, Hyde, \& Allan, 2001; Wolters, Wensing, Van Weel, Van der Wilt \& Grol, 2002). Based on evidence from epidemiologic and clinical studies, researchers have suggested that perceived severity or degree of bother is a primary trigger for help seeking. Specifically, the higher the degree of bother or perceived severity, the more likely an individual is to seek help (Andersson et al.; Macfarlane et al., 1995; Shaw et al.). However, Sarma et al. (2003) found that physical severity of urinary symptoms only partially predicted "bothersomeness" of symptoms in men with LUTS. Even though some men reported an increase in symptom frequency, this did not necessarily correspond to an increase in bother ratings. Based on these findings, Sarma and colleagues suggest that severity and bother do not neatly correspond, but the
\end{abstract}

Corresponding Author: Elizabeth M. Botelho, New England Research Institutes, 9 Galen St., Watertown, MA 02472, USA, ebotelho@neriscience.com.

Declaration of conflicting interests: The authors declared no conflicts of interest with respect to the authorship and/or publication of this article. 
reasons for this are not entirely known. If symptom severity alone does not engender respondent bother in a straightforward manner, then what can explain this relationship?

Theoretical models of help-seeking in health psychology posit "appraisal" of symptoms as a key process in effecting the movement from symptom experience to perception of a health threat in need of medical attention. The appraisal process includes such factors as previous experience, personality, age, gender, ethnic group, and illness representations (perceptions or attributions) among others (Shaw, 1999). Perceptions or interpretations of symptoms play a role in behavior around health care (Denny, 2009; Van Tilburg et al., 2006; Wringe et al., 2009), including help seeking for urologic symptoms (Gannon, Glover, O'Neill \& Emberton, 2004; Jacobsen et al., 1993; Melville, Wagner, Fan, Katon, Newton, 2008). Researchers of some of these prior studies (Gannon et al.; Jacobsen et al.; Melville et al.) have reported that perceptions of urologic symptoms influence help seeking.

In a study of women, perceptions of the etiology of urinary incontinence (UI) varied by age and symptom severity (Melville et al.). For example, women who viewed UI as uncontrollable and who reported lack of knowledge regarding symptoms were most often older and experienced more severe UI; whereas, women who viewed UI as simply part of being a woman were most often younger. Melville and colleagues examined perceptions in an effort to understand variations in help seeking, but reported that the reason for the lack of care seeking was still puzzling. Gannon and colleagues conducted qualitative interviews with 16 White men experiencing LUTS to explore the relationship between symptom detection, symptom perception, and symptom impact and help seeking. Respondents' perceptions of symptoms were categorized in terms of four themes: uncertainty as to whether the symptoms were really a problem; the current and potential implications of their symptoms; ways of coping with symptoms; self-evaluation and comparing their own experiences with others. Uncertainty about the nature and origins of respondents' symptoms seemed to correspond to a delay in help seeking.

To our knowledge, this is the first qualitative study to explore urologic symptoms with an eye toward understanding discrepancies in symptom severity and experience of symptom bother. We have observed that varying perceptions of urologic symptoms might "moderate" symptom bother, despite parity of symptom severity across respondents. Additionally, our sample of Black, White, and Hispanic men and women is racially and ethnically diverse. This allows for gender comparisons not possible in previous studies that included only men (Sarma et al., 2003; Gannon et al., 2004) or only women (Melville et al., 2008).

The idea of causality or "normalizing" attributions was studied by Gülec, (2008), who found that non-patients (non-help-seekers) of Fibromyalgia used more normalizing explanations of their symptoms than the Fybromyalgia help-seeker group and the control group. Gülec concluded that normalization might contribute to non-help-seeking behavior. As well, Denny (2009) studied endometriosis in 30 women, conducting semistructured interviews at two different times (a year apart). He found that respondent uncertainty is evident in the diagnosis, the course, and the future of a long-term illness and concluded that the interpretation of patients' pain is central in the uncertainty they report.

The work done by Gülec (2008) and Denny (2009) serves as a foundation on which we expand in this study. We have focused on the perception of urinary symptoms as a way of understanding varying reports of bother for the urinary symptoms of the same physical severity. We hypothesized that, given the same level of physical severity, the way in which a symptom is perceived moderates the level of bother attributed to that symptom and proposed a conceptual model to visually capture this relationship (see Figure 1). Furthermore, because bother is often associated with care seeking (Andersson et al., 2005; MacFarlane et al., 
1995; Shaw et al., 2001), we proposed that these differing perceptions might also influence care seeking.

In this article, LUTS are restricted to the storage symptoms of frequency, nocturia, and urinary incontinence (Abrams et al., 2002). To better understand the factors involved in how symptoms are perceived, we explored and compared varying attributions of bother reported by respondents with the same physical symptoms. To this end, three inter-related questions were addressed: (a) Do respondents with the same physical symptoms report different levels of bother associated with these symptoms? (b) Is symptom bother qualitatively moderated by how the symptoms are perceived? And (c) what, if any, influence does this have on respondents' decision to seek care?

\section{Methods}

\section{Participants}

The Boston Area Community Health (BACH) Survey-The Boston Area Community Health $(\mathrm{BACH})$ Survey is a population-based, random sample epidemiologic survey $(\mathrm{n}=5503)$ of a broad range of urologic symptoms. A multi-stage, stratified cluster sample design was used to obtain approximately equal numbers of Black, Hispanic, and White men and women, ages 30-79. Participants included 3202 women and 2301 men; 1767 Black respondents, 1877 Hispanic respondents, and 1859 White respondents. Self-reported information regarding urologic symptoms was collected during an at-home interview, conducted from 2002 to 2005. The sampling design and study methods (McKinlay \& Link, 2007), and the prevalence and correlates of urologic symptoms (Fitzgerald, Link, Litman, Travison \& McKinlay, 2007; Tennstedt, Link, Steers \& McKinlay, 2008) have been reported previously. Participants provided written informed consent. This study was approved by the New England Research Institutes' Institutional Review Board.

Beneath the Urologic Iceberg-"Beneath the Urologic Iceberg" is a qualitative study linked to the BACH Survey, consisting of focus groups and in-depth interviews. A primary objective was to explore factors underlying the care-seeking process for urinary symptoms. Participants were randomly sampled from each of the six subgroups of the BACH sample and included individuals who reported one or more LUTS on the survey. Fifty-eight respondents participated in a total of eight focus groups. In-depth interviews were conducted with 151 respondents.

\section{Qualitative Methods}

An interview guide was developed based on a literature review and refined through the use of a total of eight focus groups (approximately 90 minutes) with 58 respondents. We compared bother reports of respondents who described the same symptoms with the same severity level to isolate and define the perceptions moderating symptom bother (see Figure 1). Below, we describe the methodological techniques for focus groups and interviews, with the goal of enhancing the fit of the conceptual framework for this sample. The focus groups were conducted to inform the development of the in-depth interview guide and to ensure that topic areas were inclusive of respondents' concerns about LUTS. Focus groups were stratified by gender and race/ethnic group and conducted by race/ethnically matched moderators. Separate focus groups were conducted in English and Spanish for Hispanic participants based on language preference. The focus group moderators followed a semistructured guide with open-ended questions posed to participants to allow for openended responses, but to provide sufficient structure to ensure that all set questions were discussed. 
Interviews-One-to-one individual in-depth interviews were conducted with 151 respondents, randomly sampling approximately equal numbers $(\sim 25)$ in each of the six subgroups (Black, White, and Hispanic men and women). Because of the sensitive nature of the subject matter, interviewers were matched by race/ethnicity and gender wherever possible. This was not, however, possible for logistical reasons for a small minority of cases. These interviews were conducted in-person and followed the carefully constructed discussion guide that was based on the initial literature review as well as emergent themes brought up during the focus groups.

Using this interview guide, 60-minute interviews were conducted by trained interviewers in participants' homes. The semistructured guide consisted of open-ended questions that allowed respondents to provide as much detailed information as they wanted and for interviewers to probe for further information as appropriate. Because this method of one-toone interviewing allowed for expanded respondent responses and explanations, we were able to gather rich data. Our interviewing strategy included elements of both Fontana and Frey's (2005) "semistructured" interviewing and "unstructured" interviewing techniques insofar as the discussion guide was composed of well thought out and specifically structured questions (corresponding with the "semistructured" interview), but also included opportunities for interviewers to add any necessary probing and further questioning appropriate with the flow and direction of the interview (corresponding with the more informal "unstructured" interview).

One focus with the resulting interview guide was to explore perceptions of and attitudes toward urinary symptoms. We took several steps to reduce foreseeable factors that could lead to respondents feeling pressured to answer in a way they thought the interviewer wanted. First, we conducted interviews in respondents' homes so they would be comfortable with their environment and surroundings. The interviewers always stated that they were not doctors, that there were no right or wrong answers, and that they were just interested in hearing about experiences. Additionally, for each interview, interviewers attempted to initially build a rapport with respondents before asking study specific questions (such as asking about work, family, and hobbies). Interviews were digitally audio-recorded and transcribed verbatim. Spanish interviews were translated into English. Data from these interviews were analyzed for this study.

\section{Qualitative Analysis}

Following a grounded theory analytic approach, two of the qualitative researchers (EB and $\mathrm{EE}$ ) read the interview transcripts to generate a "start list" of codes, a process referred to as "open coding" (Strauss \& Corbin, 1998). Transcripts were read a second time to refine the coding structure. Transcripts were then coded for main themes, such as symptoms, care seeking, and attitudes, using Atlas.ti software (www.atlasti.com). The coding process is iterative by nature, and therefore, the research team employed the "constant comparative" approach, whereby the team members met on a regular basis to compare and discuss emergent themes and corresponding text (Glaser \& Strauss, 1967). We added new codes as additional themes developed. This iterative process allowed us to assess saturation, or the point at which no new themes or codes were identified. The list of codes, definitions for each code, and inclusion and exclusion criteria were stored in a codebook. We performed secondary coding to capture data regarding respondents' descriptions of symptoms, physical severity of symptoms, and symptom bother. We restricted the analysis to the 123 respondents that reported one or more urinary storage symptoms of frequency, nocturia, and incontinence. This group included 20 Black women, 22 Black men, 20 White women, 20 White men, 22 Hispanic women, 19 Hispanic men. 
Symptom Perceptions-Based on review of the qualitative focus group data and further developed from the qualitative interview data, we identified four symptom perception groups: causal perception, relative perception, perception of uncertainty, and undefined (see Figure 1). The causal perception group included respondents $(n=44)$ who perceived their urinary symptoms to be "normal" or "explainable"; for example, as caused by fluid intake, because of a medication, or as related to normal aging. The relative perception group included respondents $(\mathrm{n}=10)$ who assessed their urinary symptoms in comparison to more physically severe symptoms or health conditions. The perception of uncertainty group included respondents $(n=10)$ who expressed concerns about the future course of their symptom(s), were unsure of the etiology of their symptom(s), or expressed that symptoms might be indicative of an unknown health condition. The undefined group included those respondents $(n=55)$ who could not be not categorized within any of the other three perception groups. All respondents were placed in one of these groups. Four respondents were categorized in two groups because they employed two perceptions, simultaneously, in viewing their symptoms.

Categorization of "bother"-We categorized bother based on the respondent's description of how bothersome the symptoms were and the accompanying attitudes toward the symptoms. Descriptions of symptom bother were derived from each respondent's narrative, and specific responses to the following questions from the discussion guide: "People can have different feelings or reactions to having bladder, pelvic, or urinary symptoms. Thinking about your symptoms and how they affect your life, how do you feel about having these symptoms? Do these symptoms bother or worry you in any way?" Based on responses, each respondent was categorized as: (a) bothered and/or worried, (b) feeling inconvenienced and/or aggravated but not bothered or worried, or (c) not bothered or worried by symptoms. A bother category of 1 was considered the highest degree of bother, as illustrated by the following quotes: "They [frequency symptoms] bother me or worry me because I'd like to be healthy, I don't want to have any of this."; "Oh, because, think about it, having to pee so often! It's a pain. It's bothersome and you have to wear something down there all the time, as if you had your period."

A bother category of 2 was considered the middle degree of bother, as illustrated by the following quote: "They're [frequency and UI symptoms] irritating. Yeah. But other than that they; mentally it doesn't bother me. No. It's something that I feel that it's come on with age and I'm alive and that's the important thing." Another example quotes is displayed below:.

I wish I didn't have them ... Do they bother me? I mean I guess not really. I mean it's the kind of thing that occasionally I'm just a little self-conscious about. But being self-conscious allows me to just feel more confident that when things are fine, things are fine.

Responses assigned to category 3 were considered the lowest degree of bother. These respondents reported that they simply deal with their symptoms or do not view the symptoms as a health concern or problem. The following are two responses in this category: “... it's not bothering me .... I'm not concerned about the bladder .... It's just something that I've accepted and that's it. And it doesn't affect me." The second response is displayed below.

I mean I have to go to the bathroom and I know I've got to pee, only pee but a little bit. Then I go back and sit back down, then I have to go back to the bathroom, then I pee a lot. You know, but that's, I think that's normal ... nothing's wrong right now ... I don't really have a problem now.

The first author (EB) and a senior research assistant independently assessed 12 transcripts. Both then assigned a bother category for each respondent. Categorizations were compared 
and differences were resolved. This process was repeated until an inter-coder agreement of $\geq$ $90 \%$ was obtained.

Symptom severity level-To account for variation in symptom severity across the same symptoms, we assigned respondents experiencing UI to high or low severity groups based on Sandvik Severity Index scores (Sandvik, Seim, Vanvik \& Hunskaar, 2000) derived from the BACH survey data, and verified this by comparing it to the degree of physical severity reported in the qualitative interviews. The Sandvik score is based on a combination of leakage frequency and amount of urine leaked and is intended to assess the physical severity of UI. For the purposes of this study, low and high severity categorizations were determined to correspond with score ranges. A Sandvik score of slight or moderate (1-4) was defined as a low severity, and a score of severe (6-8) was defined as high severity (see Table 1).

Because there is no comparable measure of severity for urinary frequency and nocturia, we used respondent descriptions of their symptom to assign a severity level, based on the reported number of voids during the day (frequency) or voiding at night (nocturia). The resulting severity levels for frequency were categorized as "slight" for those who voided one to four times per day, "moderate" for those who voided five to nine times per day and "severe for those who voided 10 or more times per day. For respondents who did not report daytime number of voids, we used the relevant qualitative data to assign a severity category. We then dichotomized these categories, collapsing the "slight" or "moderate" categories to low severity and the "severe" category as high severity. Reports of nocturia were treated similarly. Severity levels for nocturuia were categorized as low severity for those who voided one to two times per night and high severity for those who voided three or more times per night.

\section{Respondents who experienced more than one symptom were assessed for both} symptoms: Two researchers (EB and EE) performed the inter-coder reliability process for symptom severity scoring. They separately assessed 12 transcripts and, as previously described, used the Sandvik scores (for UI) and scoring parameters (for frequency and nocturia) to assign each to either a high or low severity level. Variations in these assignments were discussed before a final decision was reached. Again, the process was repeated until an inter-coder agreement of $\geq 90 \%$ was reached.

\section{Results}

\section{Symptom Perceptions}

Causal perception-Respondents in this group perceived their urinary symptoms to be "normal" or "explainable". Use of diuretic medications and fluid intake were often mentioned as a cause of urinary symptoms. As one respondent explained, “... I drink eight glasses of water a day and when you drink a lot of water you have to go to the bathroom." Other respondents perceived their symptoms to be a normal result of aging. One woman noted that urine leakage was, "natural in people after a certain age;" further stating that, when people get older, the muscles get stretched out, and this tends to cause urine leakage. She believed this was a common occurrence in people her age. Sometimes, respondents described their symptoms as "normal" because they knew others who had experienced the same symptom. Consistent with previous research on the "normalizing" phenomenon (Gülec, 2008; Gülec, Sayer \& Yazici Gülec, 2007), these respondents seemed to derive comfort from the knowledge that others were dealing with the same problem. This feeling of being like others, being "normal" or being able to control symptoms (e.g. that once they stopped drinking so much water/fluid or stopped taking a certain medication they would not have to void as much), often promoted a more positive attitude toward symptoms. 
Respondents in the causal perception group tended to be less bothered by their urinary symptoms when compared to respondents with the same physical symptoms in the uncertain perception and no perception groups. In this group, $62 \%$ of respondents with low severity symptoms reported low bother, as might be expected. However, $68 \%$ of those with high severity symptoms also reported low bother (see Table 2). For many of these respondents, a causal symptom perception seemed to moderate attitudes toward their symptoms and their perceived bother. For example, one respondent reported the she believed her frequent urination was because of: "The fluids I take and the type of fluids I take in, the time that I take in these fluids, and I believe not exercising consistently." She had a causal perception. Later in the interview, the same respondent, when asked if her frequent urination bothered her, answered, "No, because I think I've got the answer as to why it's happening."

Relative Perception-Respondents in this group considered their urinary symptoms in relation to other health conditions or symptoms. They tended to be less bothered by their urinary symptoms when compared to respondents with the same physical symptoms in the uncertain perception and undefined groups. These respondents tended to focus on the other more physically severe symptom or condition, rather than on their urinary symptoms. One respondent experiencing erectile dysfunction reported that his inability to get an erection and satisfy his wife was much more troubling to him than his frequent urination. When asked how serious each condition was to him on a scale from 1 to 10 , he answered, "About a 10" for the sexual dysfunction and for the frequent urination, "That's easy; 2 or 3, about .... I don't think about it. I know that it's routine, that I'm not going to get better." Another respondent placed his urinary symptoms in a hierarchy of medical conditions, explaining, "So the worst thing was the aortic valve blowing out acutely. And the second thing is the Coumadin I have to take every day. And then the last thing is the bladder ones." The frequent urination was the lowest on his list of health concerns, and his bother was rated a 3 (the lowest degree of bother).

Respondents who assessed their symptoms relative to a prior symptom or condition often had a more positive outlook regarding their urinary symptoms and were often not as bothered by these symptoms as were those who were not comparing their urinary symptoms to another, current or past, symptom or condition. In many cases, they reported being grateful and glad that they were no longer experiencing their other symptom or condition. One woman who previously had cervical cancer stated "I feel full of life, and overall, I'm fine, I feel fine, if I'm going to get sick [regarding urinary symptoms] later, I'll get sick. But I don't worry about it." After going through chemotherapy and radiation and revealing that when she had initially found out her cancer diagnosis her first words were "What day am I going to die?", one woman explained that in comparison, "the urinary one [her frequent urination issue] hasn't been much of a problem for me." When a number of the respondents reported low levels of bother associated with current urinary symptoms, they were often comparing those symptoms to their past experiences. As with the causal perception group, most respondents $(80 \%)$ in the relative perception group with low severity symptoms reported low bother, as would be expected. Again, similar to the causal perception group, many respondents (57\%) in this group with high severity symptoms also reported low bother. For many of these respondents, a relative symptom perception seemed to moderate attitudes toward their symptoms and their perceived bother.

Perception of uncertainty-These respondents expressed concerns about the future course of their symptom(s), were unsure of the etiology, or expressed concern that symptoms might be indicative of an unknown health condition. A number of these respondents worried that they might start having "accidents" (leaking urine) in the future, and that they would smell of urine. For example, one respondent stated, “... what really affects me most is that I think 'what if this gets worse?' ... . It's going to be uncomfortable 
... it might smell bad, my clothes will be wet and that type of thing." Other respondents with an uncertain perception did not know why they were experiencing their current symptoms. One respondent noted, "They [the UI symptoms] bother me and they worry me because I don't understand why I have them." This respondent's uncertainty seemed to influence her perceived degree of bother, which was 1 (the highest degree of bother).

Respondents in this group tended to be more bothered by their symptoms in comparison to respondents with the same physical symptoms in the causal perception, relative perception, and undefined groups. These respondents often feared that their symptoms could progress, which added an element of worry to their bother. One man experiencing frequent urination and nocturia stated,

I look at it as ... a disease that has to be treated or that sometimes makes me worry about the future. In the sense that in the future this might cause something that is too late or too serious to treat ... And, so far I haven't done anything to take control of this. But, yes, it worries me, and that is basically what intrigues me most. The worry of looking toward the future: if this is going to get worse.

The uncertainty about how his symptoms might progress affected his degree of worry and bother. Another respondent worried that his symptoms could be indicative of something more serious: "I think about it [the frequent urination and nocturia] in the sense I'm saying could something be going wrong there. Do I have cancer? ... Could there be a cancer?" Respondents who did not understand the reason for their urinary symptoms typically reported this as a cause for concern. Unlike the causal perception and relative perception groups, a perception of uncertainty was linked to more bother for symptoms of the same severity. In this group, none of the respondents with high severity symptoms reported low bother; rather just the opposite, $86 \%$ of respondents with low severity symptoms and a perception of uncertainty reported high bother (see Table 2). For many of these respondents, a perception of uncertainty seemed to moderate attitudes toward their symptoms and their perceived bother, such that symptoms of low severity were perceived as more bothersome than would be expected.

Undefined-These respondents did not view symptoms causally, relatively, or with uncertainty. For those in this group who had symptoms of high severity, the vast majority $(81 \%)$ reported mid-range bother or greater. This finding lends support to the moderating role of perceptions. This finding contrasted with the observations we made from the three other perception groups, where those within the causal and relative group generally reported lower levels of bother and those within the uncertain group generally reported higher levels of bother.

\section{Implications for care-seeking}

Among all non-care-seekers, across all levels of severity and bother, approximately half (48\%) belonged to the causal perception group. Many of these respondents felt their symptoms were normal or controllable. Respondents within the causal group were less likely to seek care than respondents in other groups. That is, $54 \%$ of respondents in this group sought care for their symptoms as compared to more than $70 \%$ of respondents in the relative $(75 \%)$, uncertain $(75 \%)$, and undefined (71\%) groups reported having sought health care for their symptoms.

\section{Discussion}

In this qualitative study of a diverse sample of men and women with LUTS, we found that reports of symptom severity and bother did not correspond directly. We identified three 
perceptions which moderated symptom bother: causal perception; relative perception; perception of uncertainty. Again, as Figure 1 depicts, respondents who viewed symptoms causally or relatively tended to report a lower degree of bother than those with the same symptoms but different perceptions. Respondents with a perception of uncertainty tended to report a higher degree of bother than those respondents with the same symptoms who did not view symptoms with uncertainty.

Several limitations of this study should be noted. As previously mentioned, the retrospective nature of the data did not allow us to examine how symptom perceptions might have affected care-seeking among those respondents who had previously sought care. Second, this is a qualitative study with the type of data and a sample size that did not allow for statistical comparisons. Because there is a possibility that LUTS can cause shame and/or embarrassment, to avoid bias, it is important to ensure that the study respondents are not limited to those who feel less shame, embarrassment, or stigma and are therefore more comfortable discussing potentially embarrassing experiences. We did not compare our respondents to those who were randomly selected but refused to participate in the study. However, we recently reported that respondents in this study did in fact feel a high degree of embarrassment and stigmatization (Elstad, Taubenberger, Botelho \& Tennstedt, 2010), indicating that our sample is not limited to individuals who felt no shame, embarrassment, or stigmatization.

This study has a number of strengths. The relatively large sample size allowed us to reach thematic saturation (the point at which no new themes arise in subsequent interview transcripts). Additionally, because of the use of the one-to-one interviewing and semistructured discussion guide strategies, we were able to acquire detailed explanations of respondents' experiences. Because of the interviewing technique that we used, we were able to solidify our conceptual framework and feel confident that the framework fits not only the experiences of these respondents but also might fit other conditions or illnesses as well. While other researchers (Denny, 2009; Gannon et al., 2004; Melville et al., 2008) have looked at the effect of perceptions of symptoms on individuals, we identified and compared a set of perceptions of symptoms that have not been considered together or compared with each other. In addition to a relatively large sample for a qualitative study, our study includes respondents from six racial/ethnic and gender groups, whereas previous studies of perceptions of symptoms have reported on a small sample and/or included only one gender (Gannon et al.; Melville et al., Sarma et al., 2003).

Our study has implications for care-seeking for urinary symptoms. Of importance, a greater proportion of respondents in the causal perception group had not sought health care for their symptoms, suggesting that respondents who perceived that they knew the cause of their symptoms were not as inclined to seek care for them. Analysis of this kind is limited by the retrospective nature of our data. Symptom perceptions are likely shaped by respondents' prior care-seeking. For example, a causal perception of symptoms in someone who has already sought care might be because of a healthcare provider's assurance that the symptom is "normal'. Reports from respondents who did not seek care are more telling because in these cases symptom perceptions are not shaped by a medical encounter, and those reports might have some impact on future care-seeking.

Future researchers could focus more specifically on the questions that arise when considering our framework. For example, what are the specific implications associated with individuals who view their symptoms relatively? Does it mean that people with greater disease burden already suffer additional pain/bother more readily? Are these particular individuals less likely to seek care and receive treatment for LUTS, shouldering disproportionate disease burden? Based on our sample size $(\mathrm{N}=123)$, we might be able to 
conclude that interventions aimed at improving care seeking among individuals with LUTS should be informed by our framework.

Future researchers could also investigate changes over time in symptom perceptions. Kaimal and Beardslee (2010) looked at changes in children's perceptions regarding parental depression. He categorized perceptions within three different categories: "self-oriented perspectives", "ambivalent perspectives", and "other-oriented perspectives" and found that individuals' perceptions did sometimes change depending on whether they were in a "highadversity family" or "low-adversity" family. In a similar way, future research could be conducted to identify changes in the respondents' LUTS perceptions. Furthermore, based on our data, we have suggested that perceptions of symptom severity "moderates" symptom bother; however, our analysis is qualitative and did not quantify this relationship or establish "statistical" moderation. The quantification of this relationship is one obvious "next step" suggested by our findings.

Health practitioners should be aware that patients with a causal perception might be normalizing symptoms (Gülec, 2008; Gülec, et al., 2007) and might be less likely to seek treatment because of a feeling that LUTS are a part of aging or because others experience similar symptoms. We could, therefore, make an argument for including specific questions about symptom burden and self-management in routine office visits. Health practitioners should also provide patients with treatment options, because patients with a causal perception might be carrying (and normalizing) urinary symptom burden needlessly. Individuals with a relative perception might be of particular concern, because these individuals might not recognize their symptoms as bothersome in relation to other more serious symptoms or conditions in their lives. These individuals are likely to be experiencing undue symptom burden. As urinary symptoms are often treatable both with medication and with self management strategies (Diokno, Sand, Macdiarmid, Shah \& Armstrong, 2006; Milne, 2008; Ricci et al., 2001; Skoner, 1994), alleviating these symptoms in the presence of other more worrisome health conditions might improve health-related quality of life.

\section{Acknowledgments}

We acknowledge the NIH for supplying the funding for this study (Grant No. DK073835). We also acknowledge Journel Joseph for his assistance with data analysis, and Cecilia Baker for her programming support throughout this project.

Funding: The authors disclosed receipt of the following financial support for the research and/or authorship of this article: This research was supported by a grant from the National Institutes of Health, National Institutes of Diabetes, Digestive and Kidney Disorders, Grant No. DKO73835

\section{References}

Abrams P, Cardozo L, Fall M, Griffiths D, Rosier P, Ulmsten U, et al. Wein A. The standardisation of terminology of Lower Urinary Tract function: Report from the Standardisation Sub-Committee of the International Continence Society. American Journal of Obstetrics and Gynecology. 2002; 187:116-126.10.1016/S0090-4295(02)02243-4 [PubMed: 12114899]

Andersson G, Johansson J, Sahlberg-Blom E, Pettersson N, Nilsson K. Urinary incontinence - why refraining from treatment? A population based study. Scandinavian Journal of Urology and Nephrology. 2005; 39(4):301-307.10.1080/00365590510031129 [PubMed: 16118105]

Brittain K, Perry S, Williams K. Triggers that prompt people with urinary symptoms to seek help. British Journal of Nursing. 2001; 10(2):74-76, 78, 80. [PubMed: 12170504]

Denny E. I never know from one day to another how I will feel: pain and uncertainty in women with endometriosis. Qualitative Health Research. 2009; 19(7):985-995.10.1177/1049732309338725 [PubMed: 19470614] 
Diokno A, Sand P, Macdiarmid S, Shah S, Armstrong R. Perceptions and behaviours of women with bladder control problems. Family Practice. 2006; 23:568-577.10.1093/fampra/cml018 [PubMed: 16731545]

Elstad E, Taubenberger S, Botelho E, Tennstedt S. Beyond Incontinence: the stigma of other urinary symptoms. Journal of Advanced Nursing. 2010; 66(11):2460-2470.10.1111/j. 1365-2648.2010.05422.x [PubMed: 20735505]

Fitzgerald M, Link C, Litman H, Travison T, McKinlay J. Beyond the lower urinary tract: the association of urologic and sexual symptoms with common illnesses. European Urology. 2007; 52(2):407-415.10.1016/j.eururo.2007.03.014 [PubMed: 17382458]

Fontana, A.; Frey, J. The interview: from neutral stance to political involvement. In: Deniz, N.; Lincoln, Y., editors. The Sage handbook of qualitative research. 3rd. Thousand Oaks, California: Sage Publications; 2005. p. 695-727.

Gannon K, Glover L, O'Neill M, Emberton M. Men and chronic illness: a qualitative study of LUTS. Journal of Health Psychology. 2004; 9:411-420.10.1177/1359105304042350 [PubMed: 15117540]

Glaser, BG.; Strauss, AL. The discovery of Grounded Theory: strategies for qualitative research. Chicago: Aldine Publishing Company; 1967.

Gülec H. Normalizing attributions may contribute to non-help-seeking behavior in people with fibromyalgia syndrome. Psychosomatics. 2008; 49:212-217.10.1176/appi.psy.49.3.212 [PubMed: 18448775]

Gülec H, Sayar K, Yazici Gülec Y. The relationship between psychological factors and health careseeking behavior in fibromyalgia patients. Turk Psikiyatri Derg. 2007; 18(1):22-30. [PubMed: 17364265]

Herschorn S, Gajewski J, Schulz J, Corcos J. A population-based study of urinary symptoms and incontinence: the Canadian Urinary Bladder Survey. BJU International. 2008; 101(1):5258.10.1111/j.1464-410X.2007.07198.x [PubMed: 17908260]

Jacobsen S, Guess H, Panser L, Girman C, Chute C, Oesterling J, Lieber M. A population-based study of health care-seeking behaviour for treatment of urinary symptoms. The Olmsted County Study of Urinary Symptoms and Health Status Among Men. Archives of Family Medicine. 1993; 2:729_ 735. [PubMed: 8111497]

Kaimal G, Beardslee WR. Emerging adulthood and the perception of parental depression. Qualitative Health Research. 2010; 20(9):1213-1228.10.1177/1049732310371625 [PubMed: 20530404]

Kaplan S, Roehrborn C, Chapple C, Rosen R, Irwin D, Kopp Z, et al. Mollon P. Implications of recent epidemiology studies for the clinical management of lower urinary tract symptoms. BJU International. 2009; 103(3):48-57. [PubMed: 19302502]

Kupelian V, McVary K, Kaplan S, Hall S, Link C, Aiyer L, et al. McKinlay JB. Association of lower urinary tract symptoms and the metabolic syndrome: results from the Boston Area Community Health Survey. Journal of Urology. 2006; 182(2):616-625.10.1016/J.Juro.2009.04.025 [PubMed: 19539955]

Macfarlane G, Sagnier P, Richard F, Teillac P, Botto H, Boyle P. Determinants of treatment-seeking behaviour for urinary symptoms in older men. British Journal of Urology. 1995; 76(6):714-718. [PubMed: 8535714]

McKinlay JB, Link CL. Measuring the urologic iceberg: design and implementation of the Boston Area Community Health (BACH) Survey. European Urology. 2007; 52:389-396.10.1016/j.eururo. 2007.03.013 [PubMed: 17383808]

Melville J, Wagner L, Fan M, Katon W, Newton K. Women's perceptions about the etiology of urinary incontinence. Journal of Women's Health. 2008; 17(7):1093-1098.10.1089/jwh.2007.0606

Milne JL. Behavioral therapies for overactive bladder: making sense of the evidence. Journal of Wound Ostomy and Continence Nursing. 2008; 35(1):93-101.

Pinnock C, Marshall VR. Troublesome lower urinary tract symptoms in the community: a prevalence study. Medical Journal of Australia. 1997; 167(2):72-75. URL: http://www.mja.com.au/> (C) 1997 Medical Journal of Australia. [PubMed: 9251691]

Ricci J, Baggish J, Hunt T, Stewart W, Wein A, Herzog A, Diokno A. Coping strategies and health care-seeking behavior in a US national sample of adults with symptoms suggestive of overactive bladder. Clinical Therapeutics. 2001; 23(8):1245-1259. [PubMed: 11558861] 
Sandvik H, Seim A, Vanvik A, Hunskaar S. A severity index for epidemiological surveys of female urinary incontinence: comparison with 48-hour pad-weighing tests. Neurology and Urodynamics. 2000; 19:137-145.

Sarma A, Wei J, Jacobson D, Dunn R, Roberts R, Girman C, et al. Jacobsen S. Comparison of lower urinary tract symptom severity and associated bother between community-dwelling black and white men: the Olmstead County Study of Urinary Symptoms and Health Status and the Flint Men's Health Study. Urology. 2003; 61(6):1086-1091.10.1016/S0090-4295(03)00154-7 [PubMed: 12809866]

Shaw C. A framework for the study of coping, illness, behaviour and outcomes. Journal of Advanced Nursing. 1999; 29(5):1246-1255. [PubMed: 10320510]

Shaw C, Tansey R, Jackson C, Hyde C, Allan R. Barriers to help seeking in people with urinary symptoms. Family Practice. 2001; 18:48-52. [PubMed: 11145628]

Skoner MM. Self-management of urinary incontinence among women 31 to 50 years of age. Rehabilitation Nursing. 1994; 19(6):339-343. 347. [PubMed: 7855400]

Strauss, AL.; Corbin, J. Basics of qualitative research Techniques and procedures for developing Grounded Theory. Thousand Oaks: Sage Publications; 1998.

Tennstedt S, Link C, Steers W, McKinlay J. Prevalence of and risk factors for urine leakage in a racially and ethnically diverse population of adults: the Boston Area Community Health (BACH) Survey. American Journal of Epidemiology. 2008; 167(4):390-399.10.1093/aje/kwm356 [PubMed: 18182376]

Van Tilburg M, Venepalli N, Ulshen M, Freeman K, Levy R, Whitehead W. Parents' worries about recurrent abdominal pain in children. Gastroenterology Nursing. 2006; 29(1):50-55. [PubMed: 16552300]

Wolters R, Wensing M, Van Weel C, Van der Wilt G, Grol R. Lower urinary tract symptoms: social influence is more important than symptoms in seeking medical care. BJU International. 2002; 90:655-661.10.1046/j.1464-4096.2002.02996.x [PubMed: 12410742]

Wringe A, Roura M, Urassa M, Busza J, Athanas V, Zaba B. Doubts, denial and divine intervention: understanding delayed attendance and poor retention rates at a HIV treatment programme in rural Tanzania. AIDS Care. 2009; 21(5):632-637.10.1080/09540120802385629 [PubMed: 19444672]

\section{Biographies}

Elizabeth M. Botelho, MS, is a Senior Research Associate in the Center for Qualitative Research at New England Research Institutes, Watertown, MA, USA.

Emily A. Elstad, MPH, was a Project Manager in the Center for Qualitative Research at New England Research Institutes, Watertown, MA. She is now a doctoral student at University of North Carolina at Chapel Hill, Chapel Hill, NC, USA.

Simone P. Taubenberger, PhD, was a Research Scientist in the Center for Qualitative Research at New England Research Institutes, Watertown, MA, USA.

Sharon Tennstedt, PhD, is Vice President, Behavioral and Social Sciences, at New England Research Institutes, Watertown MA, USA. 


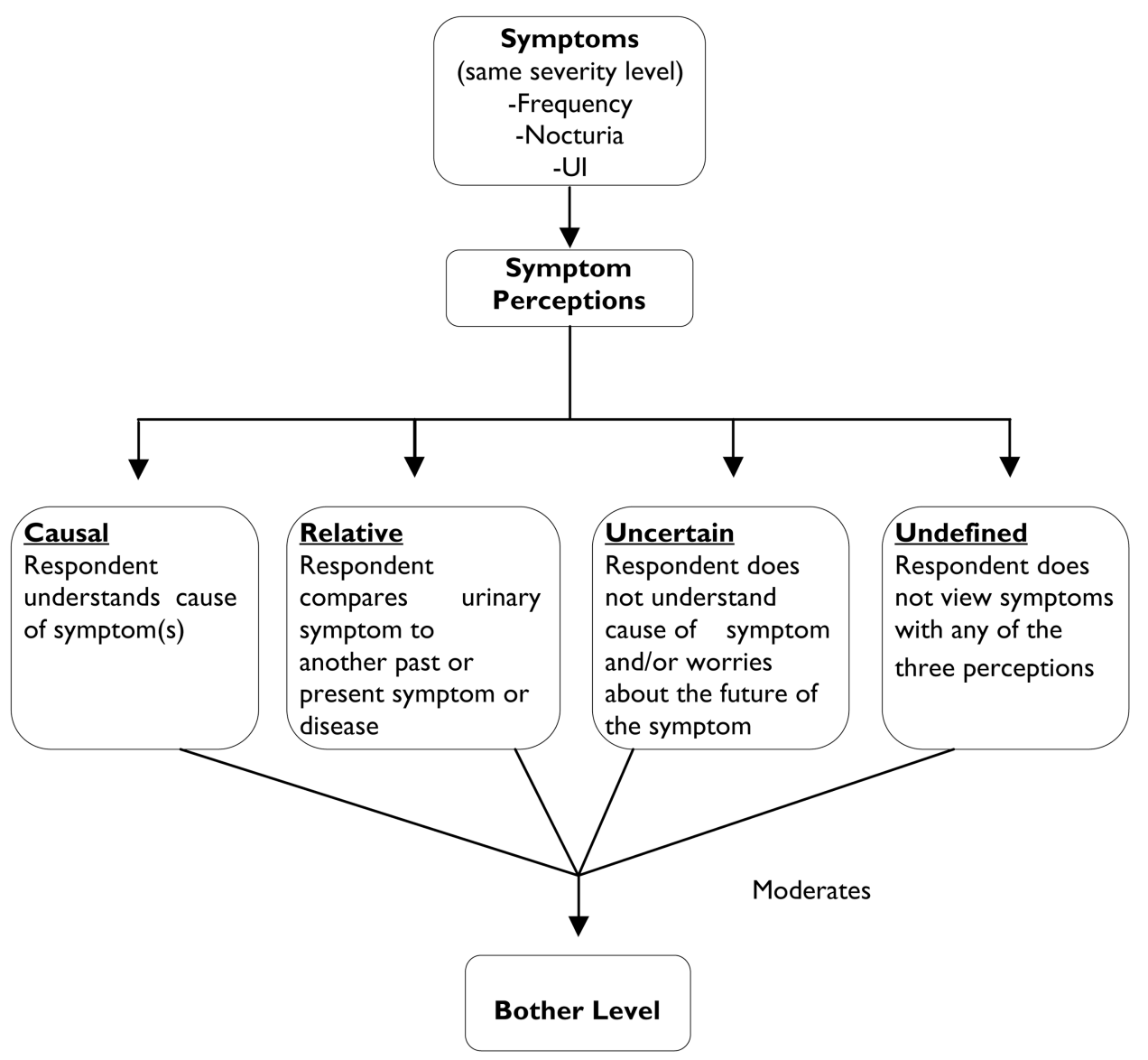

Figure 1. Conceptual framework 
Table 1

Recode of Sandvik Severity Index Scores into Dichotomous Categories

\begin{tabular}{lcc}
\hline \multicolumn{1}{c}{ Frequency } & \multicolumn{2}{c}{ Amount } \\
& Drops (1) & More (2) \\
\hline (1) less than once a month & Low (1) & Low (2) \\
(2) a few times a month & Low (2) & Low (4) \\
(3) a few times a week & Low (3) & High (6) \\
(4) every day and/or every night & Low (4) & High (8) \\
\hline
\end{tabular}


